benign gastric ulcers can potentially reduce the burden of FU-OGD.

Methods All patients with a first endoscopic diagnosis of gastric ulcer between January 2012 and September 2013 at this large teaching hospital were included. Patients with known gastric ulcers prior to study period or those referred for tertiary assessment were excluded. We defined neoplastic disease as histological evidence of gastric dysplasia or malignancy. Benign disease was defined as patients with complete ulcer healing, those with 2 sets of benign biopsies and no endoscopic suspicion of malignant disease, or in cases without FU-OGD one set of negative biopsies and at least 360 days cancer free survival. Patients with insufficient follow-up were excluded. We analysed the influence of demographic, endoscopic and histological factors on the likelihood of benign disease using chi-square test for categorical and t-test for continuous variables. Independence of variables was analysed using linear regression analysis.

Results Of 377 patients included 350 (92\%) had benign disease. 19 patients were diagnosed with adenocarcinomas, 2 with dysplasia, 5 with lymphomas, and 1 with melanoma. Patient sex, indication for gastroscopy and helicobacter pylori status did not influence the likelihood of benign disease. Benign disease was significantly associated with ulcer location in the antrum ( $\mathrm{p}=$ $0.001)$, endoscopic benign appearance $(\mathrm{p}<0.001)$, non-cratered ulcer morphology ( $\mathrm{p}<0.001$ ), benign histology on 1st biopsy $(\mathrm{p}<0.001)$, younger age (64 vs 73 years, $\mathrm{p}=0.02)$, lower number of ulcers $(1.4$ vs $2, \mathrm{p}<0.001)$ and smaller ulcer size (10 vs $28 \mathrm{~mm}, \mathrm{p}<0.001)$. After linear regression analysis only endoscopic benign appearance $(\mathrm{p}=0.03)$, benign histology on 1 st biopsy ( $p<0.001)$, lower number of ulcers $(p<0.001)$ and smaller ulcer size $(p=0.004)$ were independent predictors of benign disease.

Conclusion We have demonstrated that several simple factors collected during index endoscopy and ulcer biopsy can predict benign disease. Risk stratification according to those factors could be used to re-examine the need for FU-OGD for all patients with gastric ulceration. If prospectively verified the described predictive factors could be used to identify low risk patients who do not require endoscopy. This may be a strategy to reduce the burden of FU-OGD.

Disclosure of Interest S. Thanaraj: None Declared, A. Sainsbury: None Declared, R. Cochrane: None Declared, C. Selinger Grant/ research support from: Ferring, Shire, Nycomed, Warner Chilcott.

\section{PTU-145 HOW COMMONLY IS UPPER GASTROINTESTINAL CANCER DIAGNOSED FOLLOWING AN ENDOSCOPY THAT DOES NOT REPORT CANCER (AN ANALYSIS OF 11 YEARS OF NATIONAL DATA IN ENGLAND)?}

${ }^{1} \mathrm{D}$ Cheung* ${ }^{*}{ }^{2} \mathrm{~F}$ Evison, ${ }^{3} \mathrm{P}$ Patel, ${ }^{1} \mathrm{~N}$ Trudgill. ${ }^{1}$ Department of Gastroenterology, Sandwell General Hospital, West Bromwich; ${ }^{2}$ Health Informatics Department, Queen Elizabeth Hospital; ${ }^{3}$ School of Cancer Sciences, University of Birmingham, Birmingham, UK

\subsection{6/gutjnl-2014-307263.219}

Introduction Oesophagogastroduodenoscopy (OGD) is the investigation of choice for excluding upper gastrointestinal cancer (UGIC). However, published studies suggest 14\% of UGIC subjects had an OGD that failed to diagnose cancer in the 3 years prior to diagnosis (post gastroscopy UGIC (PGUGIC)). We have investigated the rate and risk factors for PGUGIC in a national data set in England.

Methods Hospital Episode Statistics (HES) collate information on all NHS hospital attendances in England. Subjects undergoing
OGD without an UGIC diagnosis 6-36 months before subsequent diagnosis were identified as PGUGIC cases (definitely missed - OGD without UGIC diagnosis 6-12 months prior to UGIC diagnosis; probably missed - OGD without UGIC diagnosis $12-36$ months prior to UGIC diagnosis) and subjects with no OGD 6-36 months before diagnosis served as controls. The influence of personal and institutional variables on PGUGIC were examined by multivariate logistic regression.

Results HES records from 2001-2012 were analysed including 5826932 OGD in 4163023 subjects. 132075 subjects were diagnosed with UGIC. 5659 (4.3\%) definitely missed PGUGIC cases and $8518(6.4 \%)$ probably missed PGUGIC cases were found. Gastritis/duodenitis (2512 subjects, 17.7\%) and gastric ulcer (2117 subjects, 15.0\%) were the most common coded findings in PGUGIC cases. Emergency OGD was negatively associated with PGUGIC compared with day case OGD (OR 0.70 (95\% CI $0.67-0.73), \mathrm{p}<0.001)$. Female gender (1.19 (1.1-1.2), $\mathrm{p}<$ $0.001)$, South Asian $(1.32(1.2-1.6), \mathrm{p}<0.001)$ and Afro-Caribbean $(1.26(1.1-1.5), \mathrm{p}<0.001)$ ethnicity and comorbidities (liver disease $(3.05,(2.3-4.1), \mathrm{p}<0.001)$, severe liver disease (3.01 (2.1-4.2), $\mathrm{p}<0.001)$, peptic ulcer (1.98 (1.9-2.1), $\mathrm{p}<$ $0.001)$, pulmonary disease $(1.17(1.1-1.3), \mathrm{p}<0.001))$ were associated with PGUGIC. Subjects with PGUGIC were less likely to undergo surgery $(0.76(0.7-0.8), \mathrm{p}<0.001)$ or chemotherapy (0.49 (0.47-0.51), $\mathrm{p}<0.001)$ than controls, however, this did not affect overall survival, which was similar to controls. There was a fourfold variation in PGUGIC rates between units. Unit volume did not affect the rate of PGUGIC (lowest tertile volume compared with highest tertile $0.97(0.9-1.1), p=0.5)$. The annual rate of PGUGIC did not change over the study period.

Conclusion The rate of PGUGIC up to 3 year prior to UGIC diagnosis was 10.7\% in England between 2001 and 2012. PGUGIC was associated with an elective procedure, female gender, ethnicity and comorbidities. PGUGIC subjects were less likely to have surgery or chemotherapy, although there was no effect on overall survival. There were large variations in PGUGIC rates between units but no evidence of a volume effect.

Disclosure of Interest None Declared.

\section{PTU-146 THE TRINITY OF GASTRIC EMPTYING SCINTIGRAPHY, 13C ACETATE GASTRIC EMPTYING BREATH TESTING, AND REAL TIME GASTRIC ULTRASONOGRAPHY INDICATES HIGH PREVALENCE OF GASTRIC MOTOR DYSFUNCTION IN FUNCTIONAL DYSPEPSIA}

D Nastou*, E Owen, FM Jaboli, C Clayman. Institute for Minimally Invasive Gastroenterology, Royal Free London NHS Foundation Trust, London, UK

\subsection{6/gutjnl-2014-307263.220}

Introduction Gastric motor physiology can be assessed by gastric emptying scintigraphy (GES), ${ }^{13} \mathrm{C}$ breath testing (GEBT) and real time gastric ultrasound (GUS). The aim of this study was to evaluate how commonly these tests are abnormal in patients with functional dyspepsia (FD).

Methods Twenty-seven patients fulfilling the Rome III criteria for FD were enrolled in the study. All patients had a normal upper GI endoscopy and underwent standard GES using ${ }^{131} \mathrm{I}$ technetium labelled mashed potato. On a separate day, these patients underwent a combined liquid GEBT (four hour breath test protocol using $170 \mathrm{ml}$ chocolate Ensure liquid substrate $+50 \mathrm{mg}{ }^{13} \mathrm{C}$-acetate) and GUS (calculating antral area at the time of ingestion and $15 \mathrm{~min}$ after ingestion of the GEBT liquid test meal). 